\title{
UN MODELLO PER L'INSEGNAMENTO DEGLI ARTICOLI ITALIANI A STUDENTI DI LINGUE MADRI SENZA ARTICOLI
}

\author{
Martin Testa ${ }^{1}$
}

\author{
- Ma è un gatto! \\ - Non è un gatto. È il gatto. \\ Quello che m'hai buttato dentro casa l'artro \\ giorno sur catafarco der poro nonno». \\ Rugantino (1973) ${ }^{2}$
}

\section{INTRODUZIONE}

Gli studiosi Ignacio Bosque e Javier Gutiérrez-Rexach (2008: 111) affermano che una lingua si conosce veramente quando si è capaci di utilizzare in modo corretto tutte le sue categorie funzionali (cioè preposizioni, articoli, pronomi), anche se non se ne conosce tutto il lessico; infatti, nessun madrelingua è in grado di utilizzare tutte le parole della propria lingua. Tuttavia, osserva Chiapedi (2010: 53), gli articoli rappresentano «una delle aree più problematiche» nell'insegnamento dell'italiano L2 e «lo sviluppo di tale categoria linguistica è infatti molto lento nelle prime fasi di acquisizione», nonché «soggetto a fossilizzazione a livelli avanzatì. Ciò è stato messo in evidenza da diversi lavori di indagine nell'ambito dell'analisi degli errori in italiano L2 (Cozzani, 2016; De Tommaso 2020a, 2020b; Grądzka, 2016; Runić, 2012; Škevin, Maroević, 2014; Stanič, 2017; Zgrinskić, 2019).

Benché nel parlato una persona possa comunicare in modo più o meno efficace pur utilizzando gli articoli in modo erroneo, nella scrittura questo tipo di errori diventa particolarmente visibile in quanto la definitezza non può essere espressa che verbalmente (cfr. Testa, 2019: 84, 87). Questo è di particolare importanza per gli studenti universitari la cui lingua madre è priva di un tale sistema morfologico (d'ora in poi [-art]): una debole padronanza del sistema di articoli L2 potrebbe, infatti, anche minare il loro rendimento accademico (Master, 1987, 1990).

Inoltre, e soprattutto in questi studenti, il processo diventa ancora più difficile dato che si tratta di elementi linguistici «poco salienti a livello percettivo» (Chiapedi, 2010: 53; anche Hewson, 1972), ragion per cui, almeno nelle prime fasi di acquisizione, gli apprendenti tendono a omettere gli articoli in quanto ritenuti elementi 'ridondanti' (cfr. George, 1972; Trenkić, 2009).

Va tenuto presente che l'elevato numero di regole sull'uso dell'articolo descritte nelle grammatiche tradizionali sovraccarica le risorse cognitive degli apprendenti (Testa, 2021) e questo, agli occhi di alcuni studiosi, rende gli articoli 'impermeabili all'istruzione' (ad es. Doughty, Williams, 1998: 201, cit. in Chiapedi, 2010: 70). Molti altri autori, però, richiamano l'attenzione sull'importanza di un corretto uso degli articoli della L2 (Master, 1990; Chiapedi, 2010; Testa, 2021; Aurová, Martínez Córdoba, 2015 inter alia), il cui insegnamento è un compito che viene spesso trascurato oppure mal eseguito. Runić (2012: 405), per esempio, segnala che «nelle grammatiche pedagogiche non si mette in

\footnotetext{
${ }^{1}$ Università di Varsavia.

2 Festa Campanile P. (1973), Rugantino [Film], Titanus, Roma. Il dialogo tra il Principe Niccolò Capitelli (Toni Ucci) e Rugantino (Adriano Celentano) si svolge nel minuto 0:36:30.
} 
risalto la differenza tra definitezza e specificità» (d'ora in poi $[ \pm$ def $]$ e $[ \pm$ spec $]$ ), il che spesso si traduce in una sbagliata associazione tra l'articolo definito e il tratto [ + spec] da parte degli studenti di lingue madri [-art] (cfr. Ekiert, Han, 2016; Testa, 2021). Inoltre, vi è una funzione dell'articolo propria di alcune lingue romanze, quali l'italiano e lo spagnolo, che non viene pressoché mai trattata nelle aule (cfr. Testa, 2021): la sua funzione espletiva nel caso dei soggetti preverbali (Longobardi 2014/1994: 61).

L'obiettivo di questo contributo è quello di proporre un modello semplificato ${ }^{3}$ come strategia di facilitazione per l'insegnamento degli articoli italiani a studenti la cui L1 sia priva di tale categoria linguistica. Il modello qui proposto è sviluppato principalmente a partire dell'ipotesi della fluttuazione elaborata da Ionin, Ko, Wexler (2004), nonché sulla base della corretta interpretazione dell'articolo determinativo come operatore iota (ing. :operator, Chierchia, 1998) e dell'approccio euristico proposto da Testa (2021). Va precisato che questo articolo non pretende di analizzare l'uso degli articoli italiani in maniera esaustiva, bensì di ridurre il numero di regole da memorizzare per gli studenti di madrelingue prive di articoli, specie ai livelli iniziali.

Questo articolo è organizzato come segue. In $\ 2$ si prendono in considerazione le ricerche nell'ambito dell'analisi degli errori sull'uso dell'articolo da parte degli studenti di italiano L2. In $\ 3$ si trattano i concetti fondamentali della semantica degli articoli, cioè la definitezza e la specificità, e in $\ 4$ si dedica uno spazio alla questione degli articoli italiani nel contesto dei nomi che fungono da soggetti preverbali. La proposta didattica è presentata in $\ 5$, mentre la sezione conclusiva $(\$ 6)$ riporta alcune considerazioni finali.

\section{RICERCHE SUGLI ERRORI DEGLI APPRENDENTI DI ITALIANO L2}

L'uso corretto degli articoli è di particolare importanza nella scrittura in quanto contribuisce alla coesione testuale. Benché gli errori nell'uso degli articoli non ostacolino la comprensione nella lingua orale, i testi di apprendenti L2 che usano correttamente gli articoli tendono ad essere valutati con punteggi più alti rispetto a quelli che li usano in modo scorretto (Jarvis et al., 2003: 381). Ciononostante, sono tuttora pochi gli studi specifici sull'apprendimento e sull'evoluzione del sistema di articoli italiani L2, specie nel caso di studenti di madrelingua [-art] (De Tommaso, 2020a: 270) ${ }^{4}$. Vi sono, però, alcuni studi recenti condotti principalmente con apprendenti di madrelingua slava (De Tommaso 2020a, 2020b; Grądzka, 2016; Runić, 2012; Škevin, Maroević, 2014; Stanič, 2017; Zgrinskić, 2019).

Bozzone Costa (2002) ha analizzato 120 elaborati (40 per ogni livello: elementare, intermedio e avanzato) di apprendenti adulti di 27 lingue materne diverse che frequentavano i Corsi di Italiano per Stranieri (CIS) presso l'Università degli Studi di Bergamo con lo scopo di fornire una visione generale degli errori lessicali in italiano L2. La studiosa sottolinea che, diversamente dal livello di padronanza manifestato nella produzione orale, nella scrittura neppure gli studenti avanzati riflettono «una competenza native-like») (2002: 38) nel loro uso degli articoli e delle preposizioni.

All'Università per Stranieri di Perugia, Chiapedi (2010) ha esaminato le composizioni scritte e le interviste orali di 13 apprendenti sinofoni di livello A2. Prima dell'analisi, gli

\footnotetext{
${ }^{3} \mathrm{Va}$ precisato che non adopereremo qui il termine minimalista per evitare associazioni errate con il Programma Minimalista chomskiano.

${ }^{4}$ De Tommaso (2020: 270, n. 2) segnala, però, che «[p]resso la Cattedra di Lingue Romanze dell'università di Olomouc è in corso di realizzazione un corpus di testi di studenti cechi e slovacchi disponibile online e a libero accesso (progetto Czech-It!, Linguistic corpus of native Czech and Slovak learners acquiring Italian language: https://czech-it.github.io/). Lo scopo è quello di raccogliere materiale utile a studi di tipo contrastivo; non ci sono ancora analisi disponibili».
} 
apprendenti avevano seguito un ciclo di lezioni (10 ore) che «avevano come obiettivo l'insegnamento di 10 funzioni dell'articolo italiano» (ibidem: 60), tra i quali la prima menzione del referente, la ripresa anaforica, la catafora, ecc. Per quanto riguarda i test orali, Chiapedi rileva che l'incidenza di omissioni è alta $(34 \%)$ nel primo test (orale) ma «le omissioni diminuiscono drasticamente nella seconda e nella terza rilevazione dei dati, in cui esse rappresentano complessivamente il 10 e 12 per cento delle occorrenze totali» (ibidem: 62). Nei test scritti, invece, gli apprendenti sembrano utilizzare l'articolo in modo corretto solo in contesti predicativi, oppure quando l'indeterminativo compare in contesti inespecifici (ibidem: 65), il che suggerisce che l'articolo indefinito viene per lo più percepito come [-spec] piuttosto che come [-def].

D'altronde, nella loro analisi della produzione scritta di studenti croatofoni del terzo anno del Dipartimento di Italianistica dell'Università di Zara, Škevin e Maroević (2014) hanno rilevato l'uso scorretto degli articoli definiti tra gli errori più frequenti, sia nella loro omissione sia nel loro uso sovraesteso. L'uso dell'indeterminativo, invece, si è rivelato più semplice e ciò potrebbe essere attribuito al transfer positivo dei valori del numerale croato 'uno' (m. jedan, f. jedna, n. jedno), nonché dell'aggettivo indefinito 'qualcuno' (m.sg. nëkì, f.sg. nëkā, n.sg. nëkō; m.pl. neki, f.pl. neke, n.pl. neka) (2014: 133).

Dopo aver esaminato un campione di 92 testi argomentativi $(\mathrm{N}=38$; A2-C1) scritti da apprendenti di madrelingua serbo-croata ${ }^{5}$, Runić (2012: 395) attribuisce gli errori nell'uso degli articoli italiani all'assenza di articolo in serbo-croato, nonché «all'interazione di fattori morfologici, sintattici e semantico-pragmatici che convergono sull'articolo, facendone un elemento estremamente complesso da acquisire». Ciononostante, gli apprendenti sembrano utilizzare gli articoli in modo via via più corretto, sia nel caso dei determinativi $(91>92>95>96 \%)$ sia nel caso degli indeterminativi $(64>78>71>$ $92 \%$ ) (ibidem: 400). Inoltre, i dati esaminati da Runić mettono in evidenza quanto sia complesso per gli studenti padroneggiare le diverse forme dell'articolo (il libro vs. l'esame

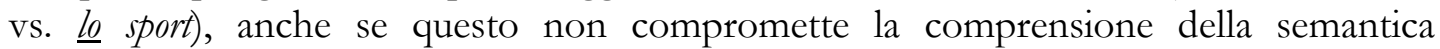
dell'articolo definito. Comunque, Runić richiama l'attenzione sull'alto numero di errori per sovrauso ${ }^{6}$ del determinativo in contesti che richiedono l'uso del partitivo, ovvero con i nomi di massa e plurali di tipo [-def, + spec], come evidenziato nei seguenti esempi (Runić, 2012: 402-3):

(1)

(a) [Alla] stazione c'è molta gente. [...] Il cibo è sul pavimento. [...] Sul pavimento sono le bottiglie, le salsicce $[\ldots]$. Ci sono dei grandi tavoli.

(A2)

(b) La stazione è sporca - i giornali sono buttati sulla terra, [...]. (B1)

(c) Un cane ha rovesciato un tavolino [...]. La tovaglia è tutta sporca e si possono vedere i piatti rotti per terra. (C1)

Per quanto riguarda lo studio di Stanič (2017: 266), la studiosa osserva che l'uso dell'articolo «si è rivelato uno dei problemi principali», a partire dall'analisi dei compiti scritti $(n=100)$ di studenti slovenofoni di Lingua e letteratura italiana presso la Facoltà di Lettere e Filosofia dell’Università di Lubiana. Nella sua analisi, Stanič (2017) rileva 397 errori vincolati all'uso degli articoli tra i quali spiccano l'uso sovraesteso dell'articolo determinativo $(\mathrm{n}=161)$, l'omissione dell'articolo $(\mathrm{n}=117)$, la forma sbagliata dell'articolo

${ }^{5}$ I dati provengono dal corpus VALICO - Varietà Apprendimento Lingua Italiana Corpus Online (Dipartimento di Studi Umanistici dell'Università degli Studi di Torino: www.valico.org).

${ }^{6}$ Probabilmente dovuto a una mancata comprensione del concetto di definitezza (Runić, 2012: 404-405). 
( $\mathrm{n}=51)$ e l'uso scorretto dell'articolo con gli aggettivi possessivi e dimostrativi ( $\mathrm{n}=40$ ). Riguardo a quest'ultimo tipo di errore, va notato che se gli studenti studiassero, invece, lo spagnolo, questo non sarebbe considerato un errore (cfr. ita. la mia macchina vs. spa. mi coche). Lo stesso vale per alcuni degli errori di forma riportati da Stanič, come *la opzione (ita. l'opzione, spa. la opción), nonché gli usi sbagliati nel caso dei membri della famiglia (ad es. ${ }^{*}$ La sua madre, ma era ${ }^{*} \varnothing$ suo ragazzo). Questi errori, pertanto, riguardano la norma dell'italiano piuttosto che la semantica dell'articolo definito.

Vale la pena menzionare che il recente studio di De Tommaso (2020a) è l'unico che si propone di analizzare gli errori vincolati all'uso dell'articolo in modo diacronico ${ }^{7}$. La sua indagine si occupa degli errori nelle produzioni scritte $(\mathrm{n}=111)$ di quattro apprendenti (due cechi e due slovacchi presso l'Università Masaryk di Brno) di italiano L2 durante il loro percorso di apprendimento dal livello elementare a quello avanzato. La ricercatrice rileva più errori nella varietà elementare, ancorché questi errori continuino «a essere preponderanti anche nei livelli successivi e riguardano in particolar modo l'articolo determinativo» (ibidem: 276), il cui uso sovraesteso viene rilevato anche al livello avanzato ${ }^{8}$. In linea con Runić (2012: 404-405), De Tommaso (2020a: 277) lo attribuisce a «una particolare incertezza» che riguarda la comprensione del tratto [ \pm def], dato che le lingue madri degli studenti «non contemplano l'uso dell'articolo». Inoltre, le indagini di De Tommaso (2020a, 2020b) sugli errori in italiano L2 da parte di studenti universitari slavofoni (cechi e slovacchi, A2-B1) mettono in evidenza che le parti del discorso più problematiche sono appunto gli articoli e le preposizioni, ovvero le categorie funzionali. Nel campione analizzato in De Tommaso (2020b), tra l'altro più ampio $(\mathrm{N}=37, \mathrm{~A} 2-\mathrm{B} 1)$, si riportano errori dovuti a una mancata percezione della semantica degli articoli (ad es. preparo la/Ø colazione; con la/Ø mia famiglia) nel primo anno $(\mathrm{N}=25, \mathrm{~A} 2)$, nonché alla difficoltà di scegliere tra determinativo e indeterminativo nel secondo anno $(\mathrm{N}=12, \mathrm{~B} 1)$.

Zgrinskić (2019) ha analizzato gli errori compiuti nell'italiano L2 scritto $(\mathrm{n}=54) \mathrm{da}$ studenti madrelingua sloveni a livello avanzato. Tra gli errori grammaticali, Zgrinskić sottolinea la frequenza di usi scorretti degli articoli e la attribuisce al fatto che lo sloveno è una lingua priva di articoli. Zgrinskić entra in dettaglio nella descrizione di questi errori e mette in rilievo che essi non si limitano a una mancata comprensione del tratto [ \pm def], ma anche alla forma degli articoli: *la amica (l'amica), *i ostacoli (gli ostacoli), *lo peso (il peso), *il angelo custode (l'angelo custode), * gli bambini (i bambini) e così via. Infatti, questo rappresenta una sfida in più per gli studenti di italiano L2, al contrario di quanto accade nell'apprendimento di lingue con articoli definiti più semplici nella loro forma, come l'inglese (the), il tedesco (der, die, das) o lo spagnolo (el, la, los, las).

D'altra parte, lo studio di Grądzka (2016) è di particolare interesse in quanto mette a confronto l'uso degli articoli da parte di studenti $(\mathrm{N}=69)$ di madrelingua polacca [-art] (italiano L2) e di madrelingua olandese [+art] (spagnolo L2). Un compito di giudizio sulla grammaticalità e un altro sull'accettabilità hanno messo in rilievo un'alta percentuale di errori di omissione dell'articolo in entrambi i gruppi, il che è da attribuirsi da un lato alla mancanza di articoli nella L1 degli studenti polacchi e dall'altro all'interferenza dei plurali generici nudi dell'olandese L1 allo spagnolo L2 (ibidem: 3). A parte questa somiglianza, i parlanti di polacco L1/italiano L2 hanno mostrato maggiori difficoltà con i sintagmi sgrammaticati nei contesti generici che in quelli specifici. Da ciò si deduce che i parlanti

\footnotetext{
${ }^{7}$ Va segnalato che nell'ambito della glottodidattica gli studi diacronici su gruppi non sono frequenti, perché si tratta di una metodologia in cui si rischia di perdere almeno una parte dei partecipanti nel tempo (ing. attrition bias). Inoltre, come osserva de Tommaso (2020a: 270), «[1]'esiguo numero di informanti non permette di poter fornire generalizzazioni definitive».

${ }^{8}$ De Tommaso (2020a: 275) osserva che, anche se il resto degli errori (lessico, ortografia, morfologia) non scompare del tutto, sono solo quelli relativi alla sintassi che tendono ad aumentare con il progredire dell'interlingua $(43,1>53,7>51,9>63,4 \%)$, dal livello elementare fino a quello avanzato.
} 
olandesi approfittano del transfer positivo del tratto [ \pm def] fra le due lingue, mentre i polacchi continuano ad associare la definitezza con la specificità. Eppure, malgrado i risultati peggiori, gli apprendenti di madrelingua polacca sembrano sviluppare una maggiore competenza linguistica nel tempo (Grądzka, 2016: 30-31):

I parlanti con un livello più alto sanno interpretare meglio dei parlanti con un livello più basso. Non si osserva lo stesso fenomeno con i parlanti olandesi. Si può quindi concludere che i parlanti nativi di una lingua senza gli articoli migliorano nell'interpretare correttamente $\mathrm{i}$ sintagmi nominali plurali raggiungendo così un livello più alto della L2. I parlanti nativi di una lingua con gli articoli invece, non migliorano, anche quando raggiungono un livello più alto.

Infine, vale la pena confrontare i risultati di queste indagini con lo studio di Cozzani (2016: 73), in quanto esamina gli errori nell'uso degli articoli italiani da parte di studenti ispanofoni ${ }^{9}$, ovvero di madrelingua [ + art], e, nonostante la vicinanza tipologica tra le due lingue, afferma che «[]]e omissioni [32,4\% sul totale di determinativi sbagliati] sono la parte più cospicua degli errori commessi negli elaborati presi in esame e anche quelle meno facili da individuare proprio perché si presentano non come un errore di forma o di uso ma come assenza dell'articolo» ${ }^{10}$. Va notato, però, che Cozzani non distingue fra sintagmi nominali oggetti e soggetti e perciò non è possibile sapere con esattezza la percentuale che riguarda gli errori per omissione nei SN soggetti.

Ciononostante, nella stragrande maggioranza dei casi è evidente l'influenza positiva dell'affinità tra i sistemi linguistici delle due lingue in questione, dato che le percentuali di uso corretto di determinativi $(97,75 \%)$ e indeterminativi (94,55\%) rappresentano un uso paragonabile a quello dei madrelingua italiani (Cozzani, 2016: 112). In sintesi, al contrario di quanto accade con gli studenti di lingue madri [-art], gli errori degli apprendenti ispanofoni si concentrano nelle differenze ortografiche (ad es. *un'altro per un altro), di genere (spa. el fin vs. ita. la fine) e di certi usi idiosincratici per interferenza della lingua madre.

Inoltre, va osservato che negli studenti ispanofoni non si riportano casi di sovraestensione dell'uso del determinativo, bensì il fenomeno opposto (2016: 116). Questi dati richiamano quanto osservato da Cominetti e Tavosanis (in stampa) in merito alla presenza di articoli nelle lingue madri degli studenti di italiano L2: quando nella L1 mancano gli articoli, gli studenti compiono più errori, specie di omissione e sovraestensione. Infatti, Cominetti e Tavosanis (in Cozzani, 2016: 118) rilevano una percentuale di errore considerevolmente più elevata negli studenti di lingua madre russa $\mathrm{e}$ ceca [-art] rispetto a quelli di lingua madre inglese, tedesca e spagnola [+art].

Questi dati ci permettono di fare alcune considerazioni importanti. La prima riguarda il fatto che gli studenti di lingue madri [-art] sembrano seguire un percorso che va dalla totale omissione ${ }^{11}$ dell'articolo, in quanto ritenuto elemento 'ridondante', al suo uso sovraesteso, fenomeno conosciuto nella letteratura in inglese con il nome di defflooding (Master, 1987), e dovuto a una erronea comprensione del tratto [ \pm def] (cfr. $\$ 3$ ).

In secondo luogo, benché la vicinanza tipologica tra le lingue [+art] costituisca perlopiù una fonte di transfer positivo, la variazione e la microvariazione fra queste lingue vanno esaminate con attenzione (cfr. $\int 3$ e $\ 4$ ). Pur trattandosi di lingue appartenenti a gruppi linguistici assai vicini, vi sono, ad esempio, parecchie differenze di uso tra gli articoli

\footnotetext{
${ }^{9}$ Sulla base di elaborati scritti raccolti nel corpus ICoN (Italian Culture on the Net).

${ }^{10}$ Inoltre, risulta che le omissioni sono più frequenti nel caso degli indeterminativi (Cozzani, 2016: 85).

${ }^{11}$ Vale anche la pena notare che le omissioni sono più frequenti nei test orali, in quanto compiti meno 'controllati 'dai parlanti (Chiapedi, 2010: 66; anche Krashen, 1982).
} 
italiani e spagnoli (cfr. Cozzani, 2016), specie per quanto riguarda i toponimi, le espressioni temporali, i sintagmi preposizionali introdotti da in (ad es. vado in ufficio/chiesa/piqzeria vs. voy a la oficina/iglesia/pizzería), certi locuzioni cristallizzate (cfr. leggere fra le righe vs. leer entre líneas), nonché i possessivi. Con questi ultimi, vale notare che l'articolo italiano ha compiuto un percorso diametralmente opposto a quello dell'articolo spagnolo, ovvero nell'italiano il suo uso è aumentato (Serianni, 2006: 178-179), mentre con i possessivi spagnoli gli articoli sono scomparsi a partire dal XV secolo (Lapesa, 1981: 281, 479). Poiché si tratta di differenze poco percettibili, queste interferenze vanno controllate affinché non si fossilizzino.

Infine, anche se non ce ne occuperemo qui, non andrebbero tralasciati gli errori che riguardano la forma dell'articolo, tra i quali *un'appartamento, *Lo sistema, *il studio - *i studi (Škevin, Maroević, 2014: 132), visto che si tratta di errori persistenti sia tra gli studenti di lingue madri [-art] sia tra quelli madrelingua [+art] e vengono riportati in pressoché tutti i suddetti studi.

\section{DEFINITEZZA VS. SPECIFICITÀ}

Da un punto di vista tipologico (si veda la Tabella $1^{12}$ ), il sistema di articoli italiani rispecchia in certo modo quello di molte altre lingue dell'Europa occidentale, costituendo così una sorta di 'lega linguistica', conosciuta con i nomi di Charlemagne Sprachbund (van der Auwera, 1998) o Standard Average European (si veda la mappa in Haspelmath, 2001: 1494; anche Grandi, 2010: \2), le cui lingue possiedono un sistema di articoli (cioè determinativi, indeterminativi più il cosiddetto articolo 'zero') che codificano la definitezza.

Tabella 1. The Nominal Mapping Parameter (NMP, adattato da Chierchia, 1998: 353-400)

\begin{tabular}{|l|l|l|}
\hline \multicolumn{1}{|c|}{ Parametri } & \multicolumn{1}{|c|}{ Caratteristiche } & \multicolumn{1}{c|}{ Esempio } \\
\hline$[+$ arg, - pred $]$ & $\begin{array}{l}\text { Gli argomenti sono SN (ogni nome è di } \\
\text { massa) } \\
\text { Non c'è marca di plurale }\end{array}$ & $\begin{array}{l}\text { Cinese } \\
\text { Giapponese }\end{array}$ \\
\hline$[+$ arg, + pred $]$ & $\begin{array}{l}\text { I sintagmi nominali possono essere } \\
\text { predicativi o argomentali } \\
\text { Argomenti nudi ammessi }\end{array}$ & $\begin{array}{l}\text { Lingue slave [-art }] \\
\text { Lingue germaniche [+art] }\end{array}$ \\
\hline$[-$ arg, + pred $]$ & $\begin{array}{l}\text { I sintagmi nominali possono essere solo } \\
\text { predicati e hanno bisogno di un } \\
\text { determinante per diventare argomenti }\end{array}$ & $\begin{array}{l}\text { Italiano }(\delta) \\
\text { Francese (senza } \delta)\end{array}$ \\
\hline
\end{tabular}

D'altronde, le lingue senza articoli utilizzano altri mezzi per esprimere la definitezza come, per esempio, l'ordine dei costituenti, in quanto i sintagmi nominali definiti si

${ }^{12}$ La variante [-arg, -pred] è impossibile, perché i sintagmi nominali non avrebbero alcuna interpretazione. 
trovano di solito in posizione preverbale, mentre quelli indefiniti compaiono perlopiù dopo il verbo ${ }^{13}$, come osservato nei seguenti esempi tratti dal cinese (Limonta, 2009: 33) e dal polacco (Pawlik, 2001: 48; Szwedek, 1976: 267):

(a) Nǐ wănshàng chī pingguǒ tu notte mangiare mele 'la sera tu mangi delle mele'

(b) Píngguǒ ň̀ jùjīng chī le mele tu già mangiato 'le mele le hai già mangiate'

(3)

(a) Na stole jest ksiażka sul tavolo c'è (un) libro

(b) Ksiażka jest na stole (il) libro è sul tavolo

(c) Kiedy wszedtem zobaczytem, że mężczyzna stat pray oknie quando sono entrato ho visto che (l')uomo era in piedi accanto alla finestra

(d) Kiedy wszeedtem zobaçytem, że prày oknie stat mężczyzna quando sono entrato ho visto che accanto alla finestra c'era (un) uomo

Qui va aperta una piccola parentesi. Anche se le lingue slave sono note per essere prive di un sistema di articoli, vi sono alcune eccezioni che vale la pena menzionare. Nel contesto della lega linguistica balcanica (ted. Balkansprachbund), il bulgaro e il macedone possiedono un articolo determinativo posposto al nome (ad es. жена 'donna', жената 'la donna'; Sussex, Cubberly, 2006: 235) e lo stesso accade in alcuni dialetti della Russia settentrionale (ad es. kníga 'libro', knígata 'il libro'; Sussex, Cubberly, 2006: 524). D’altro canto, i dialetti sloveni parlati in Italia, specie il dialetto resiano (slo. rezijanšcina) nel FriuliVenezia Giulia (Runić, 2013: 97), hanno un sistema di articoli (m. te, nt. to, f. ta) che differiscono del resto dei dimostrativi slavi nel fatto che i primi compaiono solo in contesti definiti e non in contesti indefiniti specifici (p. es. ho conosciuto una/ *la ragazad) ${ }^{14}$.

Dal punto di vista degli universali linguistici, Greenberg (1978) segnala che il ciclo evolutivo del determinativo comporta un primo stadio in cui esso compare nella lingua con un valore deittico-anaforico (come nel caso dell'evoluzione dei dimostrativi latini), un secondo stadio in cui l'articolo acquisisce il tratto [ \pm def] per essere infine ridotto a un espletivo che funge da marca nominale, come accade nel francese. Benché gli articoli germanici si trovino nel secondo stadio di evoluzione, gli articoli italiani e spagnoli sembrano appartenere ad una zona tra il secondo e il terzo stadio, in quanto l'articolo obbligatorio in posizione di soggetto preverbale va oltre il mero valore deittico-anaforico ma non è ancora del tutto «spogliato della sua funzione originaria e ridotto a mera marca nominale» (Grandi, 2010: \3).

Come risaputo, nelle lingue romanze, gli articoli determinativi rappresentano l'esito degli antichi pronomi latini ille, illa e illud. Le prime testimonianze dell'articolo

\footnotetext{
13 Tuttavia, quanto meno nel caso del cinese, «la corrispondenza tra posizione e referenza è una questione di frequenza, quindi di tendenza, e non il risultato di restrizioni sintattiche assolute» (Gottardo, Valentini, 2018: 233-234).

14 Tuttavia, Runić afferma che gli articoli resiani hanno una distribuzione molto più ristretta rispetto agli articoli romanzi e germanici.
} 
determinativo italiano risalgono al VI secolo, quando l'antico pronome latino comincia a comportarsi sintatticamente come articolo, per influenze greche (Rohlfs, 1968: 99). Gli articoli indeterminativi, invece, hanno origine dai numeri latini ünus, üna e ünum, sebbene nella lingua antica spesso non siano usati (cfr. Rohlfs, 1969: 38). In questo senso, l'acquisizione dell'indeterminativo potrebbe ritenersi più facile dato che in molte lingue, pur prive di un sistema di articoli, la cardinalità viene naturalmente codificata nel numerale uno, come nel caso del -(yi) cinese (Chiapeda, 2010: 56) e il bir turco (Erguvanh, Zimmer, 1994: 548):

(4)

(a) 一个人

yī gè rén

uno-CL-uomo

'un uomo'

(b) Bir doktoru aryorum

uno dottore.ACC cerco

'sto cercando un dottore'

Tuttavia, l'articolo indeterminativo eredita dal numerale ünus non solo la cardinalità, ma anche l'indefinitezza (ünus quidam 'certo', Renzi, 1982; cfr. Fernández Jódar, 2017).

Il dibattito sul concetto di definitez? $a$ ha suscitato l'interesse di linguisti (ad es. Hawkins, 1978; Kyriaki, 2020; Leonetti, 1999; Lyons, 1999; Ionin, Ko, Wexler, 2004), nonché di filosofi (Russell, 1905; Donnellan, 1966 inter alia). Ciò è dovuto al fatto che l'unicità può essere intesa in due modi, ovvero come 'assoluta' (ad es. il Sole) e come 'locale'. La prima è stata anche chiamata definitezza inerente (Grandi, 2010: \$1), unicità (Kyriaki, 2020), o definitezza 'debole' (DEF weak; Schwartz, 2009; Jenks, 2018); la seconda, invece, viene anche chiamata definitezza testuale (linguistica) o pragmatica (extralinguistica) (Grandi, 2010: \1), familiarità (Kyriaki, 2020), o definitezza 'forte' (DEF strong; Schwartz, 2009; Jenks, 2018). La differenza è evidente in tedesco, come si può osservare nei seguenti esempi tratti da Schwartz (2009: 41):

(5)

(a) In der Kabinettssitzung heute wird ein neuer Vorschlag vom Kanzler erwartet. 'Nella riunione di gabinetto di oggi si attende una nuova proposta dal Cancelliere'

(b) In der Kabinettssitzung heute wird ein neuer Vorschlag von dem Minister erwartet. 'Nella riunione di gabinetto di oggi si attende una nuova proposta dal ministro'

Nell'esempio (5a) quando si parla del Cancelliere si sa che ce n'è solo uno e in questi casi il tedesco utilizza la forma contratta vom, mentre il ministro dell'esempio (5b) è solo uno fra i tanti ministri del governo e quindi viene utilizzata la forma von dem, proprio perché nel contesto è identificabile dal ricevente. Comunque, come osserva Dalle Carbonare (2017: 44), ciò dipenderebbe dal contesto, dato che la forma in dem Garten ha una chiara funzione anaforica, mentre nel sintagma im Garten si intende l'attività di 'giocare in giardino' piuttosto che l'identificazione di un particolare giardino.

Vale la pena notare che la differenza tra unicità assoluta e locale è visibile anche in lingue senza un sistema di articoli: il cinese, come osserva Jenks (2018), preferisce i nomi nudi nei contesti di unicità assoluta ma tende a utilizzare i dimostrativi nei contesti anaforici, ovvero di unicità locale ${ }^{15}$.

15 Tranne in posizione di soggetto (Jenks, 2018). 
Tuttavia, nel caso dell'articolo determinativo italiano l'unicità del referente non andrebbe intesa nella sua accezione russeliana, cioè in termini assoluti, bensì nel senso donnelliano (1966) di 'unico nel contesto enunciativo' (cfr. Testa, 2021) ${ }^{16}$. Questo vale per la ripresa anaforica, la deissi, nonché per l'anafora associativa (Hawkins, 1978), ad es. Vado a scuola a parlare con la direttrice ('della scuola'), mentre per Russell (1905) il SN la direttrice sarebbe ambiguo in quanto non denota una singola persona identificabile in tutti $\mathrm{i}$ contesti. In ogni modo, per quanto riguarda l'uso del determinativo non ci dovrebbero essere ambiguità nell'interpretazione del referente da parte del destinatario, come spiega Hawkins (1978: 157, riportato anche in Piotrowska, Skrzypek, 2021: 18) ${ }^{17}$.

A livello formale, osserva Chierchia (1998), l'articolo definito è interpretato come l'operatore iota ' $'$ ', il quale, secondo la classica definizione di Russell, viene inteso come «the largest member of $X$ if there is one» (Chierchia, 1998: 346). In pratica, un sintagma nominale come $i$ cani (cioè, ‘ CANI) è interpretato come 'la più grande pluralità di cani', mentre lo stesso sintagma al singolare (cioè, il cane $=\imath$ CANE) significa 'l'unico cane (se ce n'è uno)'. Secondo Kyriaki (2020: 102), le lingue romanze e l'inglese avrebbero una struttura di tipo $\left[\operatorname{DefP}_{[, \mathrm{FAM}]}\left[[]\left[\left[\operatorname{Def}_{[, \mathrm{FAM}]}\right][]\right]\right]\right]$ in quanto non distinguono tra i due tipi di unicità e utilizzano un solo marcatore linguistico dal valore $[\underline{\mathrm{def}}]$, mentre quelle lingue come il tedesco e il greco avrebbero dei sintagmi di unicità $\left(\iota \mathrm{Ps}^{18}\right)$ dalla struttura $\left[\imath \mathrm{P}_{\mathrm{e}}[\iota]\right.$ $\left.\left[\mathrm{FamP}_{[\mathrm{e}, \mathrm{f}}[\mathrm{Fam}]\right]\right]$. Cionostante, le lingue con un sistema di articoli li utilizzano nel loro significato prototipico ${ }^{19}$, lasciando così delle interpretazioni alternative per i nomi 'nudi'. Nel caso dell'inglese, ad esempio, l'assenza di articolo prevede l'interpretazione ' $n$ ', vale a dire 'generica', per i nomi nudi (Chierchia 1998: 360) (ad es. Italians are good learners).

Secondo Chierchia (1998: 386), anche gli argomenti 'senza articoli' in italiano sono in realtà sintagmi determinati proiettati da un determinante nullo $\delta$, diversamente da quanto avviene in francese, lingua che non ammette $\mathrm{i}$ nomi nudi in quanto privo di un tale determinante (si veda la Tabella 1). Quando in posizione di oggetto, questo determinante $\delta$ viene interpretato come partitivo (ad. es. bevo sempre $\delta$ vino; mangio $\delta$ patate) nel caso dei nomi di massa e con i plurali. Tuttavia, il valore partitivo in italiano viene reso principalmente per mezzo dell'articolo partitivo: vorrei del latte; ho visto delle persone scappare (cfr. Viviani, 2011: \3). La differenza, in questo caso, sembra risiedere nel concetto di specificità, poiché, pur trattandosi sempre di contesti indefiniti, il determinante nullo sembra codificare referenti più generici, cioè [-spec], mentre l'articolo partitivo denota una certa specificità.

D'altra parte, però, i numerabili al singolare devono sempre ${ }^{20}$ essere accompagnati da un determinante espresso (esempi 6a-b), tranne quando si trovano in posizione non argomentale, ad. es. il vocativo (Caro amico, ti scrivo...), i predicativi (Lui è professore; Ti credevo amico di Maria) e nelle esclamazioni (Cavolo.) (Longobardi, 1994: 612; anche Chierchia, 1998: 396; Viviani, 2011: \5).

(6)

(a) *(Un/Il) grande amico di Maria mi ha telefonato. (Longobardi, 1994: 612)

(b) *Ho incontrato (un/il) grande amico di Maria ieri. (Longobardi, 1994: 612)

\footnotetext{
${ }^{16}$ In parole di Birner, Ward (1994: 1): «uniquely identifiable to the hearer».

${ }^{17}$ Ecco perché per la definitezza si sono anche adoperati termini come noto ('al destinatario') (cfr. Serianni, 2006: 161-162) e, in inglese, pressupossed o assumed known to the hearer (HK) (si veda Jarvis, 2002 e le referenze ivi citate).

18 Stà per iota phrase, da non confondere con i sintagmi flessivi (ing. inflectional phrase IP).

${ }^{19}$ Cioè, l'operatore iota per i definiti e il quantificatore esistenziale $\exists$ ('esiste almeno uno') per gli indefiniti (Chierchia, 1998).

20 Pure nel caso dei soggetti posverbali dei verbi inaccusativi: È venuto *(un/il) grande amico di Maria (Longobardi, 1994: 612).
} 
Naturalmente, vi sono eccezioni, specie nel caso di espressioni consolidate che andrebbero imparate a memoria, ad es. cercare casa, trovare marito, prendere cappello, ecc. (Grandi, 2010: \4), e con alcune espressioni introdotte da preposizione, ad es. da giovane, vado in ufficio, sono in sede, ecc. (Viviani, 2011: \5). Secondo Renzi (1985: 283), nei casi suddetti l'articolo zero rimuove il referente dal sintagma nominale e quindi l'interpretazione del sintagma è più vicina all'azione, anziché al referente. Inoltre, Rohlfs (1969: 34) segnala che l'articolo manca anche in numerosi nessi formati nel protoromanzo, quando la categoria non era ancora obbligatoria, ad es. tra cielo e terra, giorno e notte, marito e moglie, ecc.

Eppure, non tutte le lingue utilizzano gli articoli per codificare la definitezza. Nel samoano, ad esempio, l'aspetto più rilevante non è la definitezza ma la specificità, la quale viene codificata per mezzo degli articoli le [+spec] e se [-spec] (Ionin, Ko, Wexler, 2004). Secondo l'ipotesi della fluttuazione (Ionin, Ko, Wexler, 2004), gli apprendenti di una seconda lingua con articoli oscillano tra i valori [def] e [spec] finché non riescono ad aggiustare il parametro a seconda dei valori della lingua di destinazione. Il fatto che la specificità non sia così rilevante al momento di scegliere tra il determinativo e l'indeterminativo in italiano viene illustrato da Runić (2012: 398), in quanto i sintagmi definiti possono essere sia especifici sia inespecifici e quindi vi sono quattro possibili esiti dell'incrocio dei tratti $[ \pm$ def $]$ e $[\underline{\text { spec}}]$ :

(7)

(a) Come fai a trovare il biglietto per X? Ho un contatto. [-def, +spec]

(b) Cosa vorrebbe fare quando si sposa? Avere un figlio. [-def, -spec $]^{21}$

(c) E cosa farà con il figlio quando andrà a vivere negli Stati Uniti? [+def, +spec]

(d) $\{$ Il / Un $\}$ cane è un mammifero. I cani sono mammiferi. [+def, -spec]

Come abbiamo già visto nel $\ 2$, nelle ricerche sull'acquisizione degli articoli L2 è stata registrata una fase iniziale in cui l'articolo viene omesso (in quanto elemento 'ridondante') per poi passare a un uso sovraesteso del determinativo che mette in evidenza l'errata associazione tra l'articolo e il valore [+spec]. Questo fenomeno, conosciuto con il nome di defflooding ${ }^{22}$, è stato osservato negli apprendenti di inglese L2 (Ekiert, Han, 2016; Ionin, Ko, Wexler, 2004; Master, 1987), di spagnolo L2 (Fernández Jódar, 2006; Rodríguez García, 2019), nonché di italiano L2 (De Tommaso, 2020a; Runić, 2012; Stanič, 2017; Škevin e Maroević, 2014; Zgrinskić, 2019). Ecco alcuni esempi:

(8)

(a) è la persona alla quale tiene molto (Zgrinskić, 2019:36)

(b) Silvia è la compagna di classe di Leo (Zgrinskić, 2019: 37)

(c) Che casino! [...]. È la situazione molto interessante. (Runić, 2012: 401; A2)

(d) All'improvviso ho sentito la voce femminile che gridava [...] (Runić, 2012: 401; C1)

(e) Mi trovo nell'aula molto grande, ci sono tante tavole (De Tommaso, 2020a: 272)

(f) Dopo [...] comincia l'era molto produttiva (De Tommaso, 2020a: 277)

${ }^{21}$ L'indeterminato inespecifico si può combinare con gli aggettivi indefiniti (forse andrebbero chiamati inespecifici?) qualsiasi o qualunque, nonché ammette essere ripreso anaforicamente utilizzando il pronome ne (ad. es. Sto cercando un libro [qualsiasi qualunque] da leggere, ma non [lo ne] trovo nessuno; si vedano altri esempi in Grandi, 2010: \1).

22 Vale la pena notare che il fenomeno opposto, ovvero l'uso sovraesteso dell'indeterminativo o del partitivo, è estremamente raro (Stanič, 2017: 268). 
È da pensare che ciò sia dovuto a spiegazioni fuorvianti oppure ad associazioni erronee dell'articolo determinativo con la specificità, spesso per via del trasferimento del valore deittico dei dimostrativi delle lingue madri [-art] degli studenti. Comunque, i dati ricavati dalle indagini nell'ambito della glottodidattica esigono un approccio diverso focalizzato sulla comprensione del concetto di definitezza, specie di quella 'locale'.

\section{LA POSIZIONE DI SOGGETTO}

A prima vista, un'istruzione volta all'acquisizione del valore [ \pm def] dovrebbe essere sufficiente per ridurre al minimo il numero di errori o, in altri termini, per aiutare gli studenti a sviluppare una sufficiente 'competenza procedurale' (Wilson, Sperber, 2012: 150) che consenta loro di utilizzare correttamente gli articoli italiani. Tuttavia, ciò che sembra vero per lingue come l'inglese (si vedano Master, 1987, 1990; Ekiert, Han 2016; Ionin, Ko, Wexler, 2004 inter alia), non lo è affatto per altre come l'italiano e lo spagnolo (cfr. Testa, 2021).

Le lingue germaniche ${ }^{23}$ utilizzano gli articoli per codificare la definitezza anche in posizione di soggetto, e perciò i soggetti generici germanici sono perlopiù nudi ${ }^{24}$. In questo senso, vale la pena menzionare che, come osserva Dalle Carbonare (2017), gli studenti italiani di tedesco sbagliano al contrario degli apprendenti di italiano L2, cioè aggiungono gli articoli in contesti che richiedono sintagmi nominali nudi, specie con $i$ soggetti preverbali, ad es. (*Die) Zeit is Geld ('Il tempo è denaro', ibidem: 136) e (*Die) Griechen und (*die) Römer legten das Fundament zu unserer Kultur ('I greci e i romani hanno posto le basi della nostra cultura', ibidem: 71$)^{25}$.

In italiano i sintagmi nominali nudi vengono ammessi solo quando governati da una testa preposizionale (Leo è andato da amici) o verbale (Leo ha mangiato patate) (Chierchia, 1998: 383). Invece, sono mal tollerati «in posizione di soggetto di una frase indipendente dichiarativa», ad es. *riso sta sullo scaffale a destra e *libri di fantascienza sono sul mio comodino (Grandi, 2010: $\left.\int 4\right)^{26}$. Sebbene i nomi nudi ricevano per lo più un'interpretazione partitiva (cfr. \3), nel caso dei soggetti i partitivi 'nudi' tendono a preferire la posizione postverbale. Infatti, insieme all'opposizione tra essere e avere (cfr. sono/*hanno piovuto critiche), l'accettabilità di soggetti nudi postverbali rappresenta uno dei diagnostici più utilizzati per determinare l'ergatività di un verbo (Mackenzie, 2006: 70; cfr. Testa, 2021). Per esprimere la partitività in posizione preverbale, però, i soggetti italiani richiedono sempre l'articolo, in questo caso il partitivo (Chierchia, 1997: 74):

(9)

(a) *(Dell') Acqua sta gocciolando dal rubinetto.

(b) *(Dei) Cani stanno abbaiando in cortile.

In altre parole, e sempre sulla base dell'esempio (9b), l'inglese distingue tra Dogs [-def] e The dogs [+def], mentre l'italiano lo fa tra Dei cani [-def] e I cani [+def] (cfr. Chierchia,

\footnotetext{
${ }^{23}$ Si veda anche Piotrowska e Skrzypek (2021) per lo svedese.

${ }^{24}$ Tranne i numerabili al singolare, ad es. The lion is a large mammal (Testa, 2021: 216), i quali tendono a utilizzare il determinativo (anziché l'indeterminativo) con i soggetti che designano unità tassonomiche, ovvero così omogenee da essere considerate una specie (Krifka et al., 1995; anche in Chierchia, 1998: 379). 25 Anche se Longobardi (1994: 653) segnala che in tedesco l'articolo con i nomi di massa e i plurali è facoltativo, l'aggiunta dell'articolo nei soggetti inglesi sarebbe sempre considerata un errore in contesti come questi.

${ }^{26}$ Lo stesso vale per lo spagnolo (si veda Testa, 2021).
} 
1998: 391). In quest'ultimo caso, la scelta fra interpretazione definita vera e propria e quella generica dipende dal tipo di predicato (si veda Krifka et al., 1995).

Un'altra prova che i soggetti in italiano richiedono la presenza di un determinante espresso si trova nella constatazione del fatto che i nomi nudi «could not appear as the small clause subject in an ECM (Exceptional Case Marking) construction» (Mackenzie, 2006: 71), come ad es. in *Consideravo [sc studenti intelligenti] (Belletti, 1988: 29).

Naturalmente, come si può apprezzare nei seguenti esempi tratti da Chierchia (1998: 384-385), capita di trovare nomi nudi nella periferia sinistra della frase ma solo in formule brachilogiche (titoli, telegrammi, avvisi), in posizione focale ${ }^{27}(10 \mathrm{a})$, in posizione distaccata a sinistra seguita dal clitico partitivo ne (10b-c), oppure in SN complessi o coordinati ${ }^{28}$ (10d-e):

(10)

(a) Pollo io voglio, non pesce.

(b) Studenti, ne ho molti.

(c) Soldi, non ne ho.

(d) Studenti *(e colleghi) hanno telefonato.

(e) Qui, ragazze in minigonna sono rare.

In sintesi, per quanto riguarda il tipo di articolo, la lingua italiana sembra distinguere tra un articolo semantico e un altro espletivo ${ }^{29}$ : il primo codifica la definitezza, mentre il secondo è inserito per soddisfare un requisito sintattico e per consentire al nome di funzionare come argomento (cfr. Kyriaki, 2020: 104). Per dirla in termini più tecnici, in lingue romanze come l'italiano e lo spagnolo (in questo senso il portoghese brasiliano sarebbe diverso, Schmitt, Munn, 2002; in Longobardi, 2014, 1994), solo i sintagmi determinati ${ }^{30}$ possono fungere da argomenti, mentre i sintagmi nominali veri e propri (ossia i nomi 'nudi') si trovano soltanto in posizioni predicative non argomentali (Longobardi, 1994). Infatti, i pochissimi numerabili al singolare in posizione di soggetto si trovano in locuzioni proverbiali quali Gallina veccbia fa buon brodo che rappresentano uno stadio anteriore nell'evoluzione dell'articolo in quanto i soggetti nei proverbi più recenti richiedono l'articolo, ad es. Il tempo è denaro (cfr. Greenberg, 1978; anche Leonetti, 1999; Kyriaki, 2020; Serianni, 2006: 184-186).

Tenuto conto di ciò, vale la pena considerare se il valore espletivo degli articoli italiani in posizione di soggetto possa essere utilizzato come un'euristica (Tversky, Kahneman, 1974; Kahneman, Klein, 2009) al fine di ridurre in modo sostanziale il numero di regole da memorizzare da parte degli studenti e così facilitare il processo di acquisizione degli articoli italiani L2. Per quanto riguarda i processi apprenditivi Kahneman (2011: 21) ci spiega che vi sono due sistemi cognitivi: uno che funziona in modo automatico e veloce

\footnotetext{
${ }^{27}$ Cfr. Rizzi (1997).

${ }^{28}$ Questo fenomeno è ciò che Suñer (1982: 209) chiama Naked noun phrase constraint, vale a dire che i nomi nudi possono fungere da soggetti preverbali purché siano accompagnati da modificatori (cfr. Grądzka, 2016: 10; Longobardi, 2001).

29 Anche l'articolo determinativo greco sarebbe espletivo (Roussou, Tsimpli, 1994), in quanto il greco non ammette soggetti nudi con valore generico (cfr. Kyriaki, 2020: 104), ad es. $\tau \alpha \dot{\alpha} \lambda \circ \gamma \alpha \alpha$ siv $\alpha \iota \omega \varrho \alpha i \alpha \zeta \dot{\omega} \alpha$ (ART.n.pl

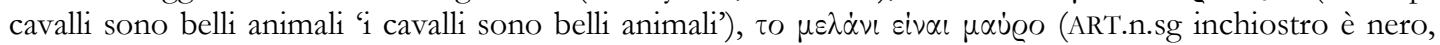
'l'inchiostro è nero') (Holton et al., 2012: 358).

${ }^{30}$ Per la differenza tra SD e SN si veda l'ipotesi originale in Szabolcsi (1983) e Abney (1987), e per la sua applicazione all'italiano cfr. Longobardi $(2014,1994)$ inter alia. In favore della universalità del sintagma determinato, anche nelle lingue slave (senza articoli) "[] posizione D deve essere sintatticamente attiva per il $N$-to-D raising che ha luogo nelle costruzioni con gli aggettivi numerali, quantificali e attributivi” (Grądzka, 2016: 11 e le referenze ivi citate).
} 
(che ci ricorda l'acquisizione come intesa da Krashen, 1982) e un altro che «allocates attention to the effortful mental activities that demand it, including complex computations», il quale ci ricorda il processo di apprendimento cosciente e razionale descritto da Krashen (1982) (cfr. Testa, 2021). Se è vero che la capacità della memoria di lavoro degli studenti è sovraccaricata dall'alto numero di regole da attivare al momento di produrre 'lingua' (Krashen, 1982), allora più regole ci sono maggiore diventa il carico cognitivo (Kormos, 1999: 218-219). In questo senso, specie ai livelli iniziali, le euristiche potrebbero essere applicate come strategie coscienti in modo da risolvere problemi complessi, come nel caso dell'uso degli articoli (Testa, 2021: 213) ${ }^{31}$.

\section{PRoposta DidATTICA}

Vale la pena ricordare che è, infatti, la salienza (insieme alla frequenza) uno dei criteri che facilitano l'acquisizione degli elementi linguistici della L2: prima si acquisiscono le parole «più brevi e semplici da pronunciare», quelle concrete e lessicali, e solo dopo quelle più complesse, quelle astratte e quelle funzionali (Rosi, Berardini, 2020: 191). In questa prospettiva, poiché gli articoli costituiscono elementi funzionali poco salienti, la semplice esposizione alla lingua di destinazione non sembra sufficiente ed è necessario richiamare l'attenzione degli studenti sulle funzioni dell'articolo italiano. Si propone, dunque, di affrontare l'insegnamento degli articoli italiani concentrandosi su due assi fondamentali, cioè la loro funzione semantica e quella espletiva, ai fini di ridurre il numero di regole da memorizzare per gli studenti di madrelingue prive di articoli, specie ai livelli iniziali.

Da un lato, la corretta scelta dell'articolo semantico comporta l'acquisizione del tratto [ \pm def], e in questo senso si dovrebbe far sì che l'attenzione degli apprendenti venga focalizzata sulla notorietà del referente dal punto di vista del ricevente, anziché dell'emittente. Questa notorietà del ricevente (o in altri termini: 'unico referente possibile nel contesto enunciativo') è fondamentale in quanto sembra coprire tutte le nozioni vincolate all'uso dell'articolo definito (anafora, endofora, catafora, unicità locale e assoluta; cfr. \3), tutti aspetti che possono essere ripresi più avanti nel percorso di apprendimento, come spunti per ulteriori riflessioni metalinguistiche.

Una strategia da evitare assolutamente è quella di insistere sulla 'concretezza' del referente come strumento di diagnosi nella scelta dell'articolo, poiché i sintagmi definiti (nonché quelli indefiniti) possono denotare nomi sia specifici sia inespecifici (cfr. ess. 7ad, supra). Questo aspetto merita speciale attenzione da parte degli insegnanti, dal momento che gli studenti di lingue madri prive di articoli si mostrano «assai propensi a impiegare il $\operatorname{dim}$ [ostrativo] al posto dell'AD» (Gottardo, Valentini, 2018: 240, e le referenze ivi citate), il che è da attribuirsi al fatto che nelle lingue [-art] i dimostrativi vengono utilizzati come marca di specificità ${ }^{32}$ ed è proprio questo valore che viene spesso trasferito agli articoli italiani L2, anziché la definitezza (cfr. \2).

D’altro canto, benché il transfer sia stato tradizionalmente percepito come negativo (cioè come interferenza), negli ultimi anni si sono sviluppati dei modelli di insegnamento plurilingue (ad es. Kucharczyk, 2018) sulla base del transfer positivo fra le lingue del repertorio linguistico dello studente. In questa prospettiva, si potrebbe approfittare della conoscenza di seconde lingue [+art], quali l'inglese, il tedesco, lo spagnolo e il portoghese,

\footnotetext{
31 Infatti, gli studenti slavi sembrano utilizzare l'euristica (nonostante sbagliata) 'articolo definito $=$ [+spec]' nell'apprendimento degli articoli romanzi (Testa, 2021: 213).

32 Come spiegato da Gottardo, Valentini (2018: 245), l'uso del dimostrativo con valore anaforico risponde a due condizioni: «il rinvio si trova a una distanza minima dalla prima menzione, cioè è immediatamente adiacente, e il referente ha forte rilevanza topicale a livello discorsivo».
} 
per riflettere sulla nozione di definitezza in quanto viene codificata in modo più o meno simile in questo tipo di lingue. Infatti, capita spesso che gli studenti credano erroneamente di trasferire il valore degli articoli L2 alla L3 quando in realtà tendono a trasferirne la 'specificità/concretezza' (cfr. Testa, 2019).

Per quanto riguarda l'articolo espletivo, invece, dagli studi sull'analisi dell'errore (\$2) si evince che gli errori per omissione dell'articolo nei sintagma nominali soggetto non possono essere attribuiti alla sola mancata acquisizione della definitezza. Infatti, si tratta di un errore che compare anche tra studenti di lingue madri [+art] (cfr. Grądzka, 2016). In questo senso, varrebbe la pena insegnare l'uso dell'articolo come requisito sintattico dei sintagmi nominali che fungono da soggetto, specie nelle prime fasi di acquisizione. Si potrebbe, per esempio, confrontare enunciati del tipo $i$ cani sono belli / $i$ cani stanno bene, precisamente per riflettere sul fatto che la specificità dei referenti andrebbe cercata nel tipo di predicato (cfr. Krifka et al., 1995), anziché negli articoli, diversamente da quanto accade nelle lingue germaniche. Si potrebbe altresì far notare che sebbene si possano trovare a volte nomi nudi nella cosiddetta periferia sinistra della frase, si tratta quasi sempre di espressioni marcate (cfr. ess. 10a-e, supra) (questi aspetti andrebbero esaminati in dettaglio ai livelli intermedi-avanzati).

Infine, le discussioni (sebbene importantissime) che riguardano la norma dell'italiano, quali l'uso e omissione dell'articolo con i nomi di parentela e altri usi idiosincratici (cfr. Serianni, 2006: 161-189), andrebbero rimandate a fasi successive del processo di apprendimento, una volta padroneggiate le funzioni semantiche ed espletive dell'articolo (ciò permetterebbe agli studenti di costruire la referenza in modo comprensibile all'interlocutore).

\section{CONCLUSIONI}

Questo contributo ha voluto proporre un modello d'insegnamento degli articoli italiani rivolto in particolare a studenti la cui lingua madre sia priva di una tale categoria grammaticale. Questo modello ha tenuto conto degli errori rilevati nelle richerche sull'italiano L2 (cfr. \2) e si è sviluppato alla luce delle teorie linguistiche attuali sulla semantica degli articoli (cfr. \3), nonché sulla loro funzione espletiva in italiano (cfr. \4).

Da quanto suggerito nella letteratura (cfr. $\int 2$ ), negli studenti di madrelingue [+art] l'acquisizione dell'articolo seguirebbe un percorso diverso rispetto agli studenti di madrelingue [-art]: sebbene i primi usino gli articoli fin dai livelli iniziali, i secondi, invece, tendono ad omettere gli articoli all'inizio in quanto ritenuti ridondanti per poi utilizzarli in modo sovraesteso, il che è da attribuirsi a una sbagliata comprensione del determinativo come $[+\mathrm{spec}]$. In questa prospettiva, il modello qui proposto è in linea con quanto suggerito da Runić (2012: 405) sull'elaborazione di "strategie che insistano sulla prospettiva dell'ascoltatore", cioè sull'insegnamento della definitezza e non della specificità.

Dal punto di vista tipologico, abbiamo visto che l'italiano utilizza gli articoli come mero requisito sintattico accanto ai nomi che fungono da soggetti preverbali ( $(3)$. In questa prospettiva, dunque, l'approccio euristico 'soggetti preverbali + articolo' potrebbe ridurre la percentuale di errori di omissione, nonché il carico cognitivo degli studenti madrelingua [-art], poiché essi avrebbero meno regole da memorizzare.

Cionondimeno, siamo consapevoli di quanto sia complesso il processo di acquisizione degli articoli italiani e, a questo riguardo, condividiamo in pieno la riflessione di Chiapedi (2010: 70) sull'importanza della sistematicità nell'insegnamento di aspetti linguistici così complessi, com'è l'uso degli articoli: 
Non tutto può e deve essere insegnato nello stesso momento: è infatti appurato che gli apprendenti, nelle prime fasi di acquisizione, non hanno il grado di maturazione psicolinguistica per processare alcuni elementi di grammatica [...]. Per questo è importante ricordare che il lavoro non si può esaurire in poche lezioni ma deve essere portato avanti ciclicamente, mantenendo un percorso necessariamente "a spirale", per esempio suddividendo le funzioni dell'articolo in base alle categorie relative al suo uso.

Infine, nonostante le difficoltà siano maggiori per gli studenti di L1 [-art], i dati ricavati da Cozzani (2016) dimostrano che l'uso dell'articolo italiano L2 non rispecchia quello di un madrelingua nemmeno quando la L1 degli studenti è tipologicamente molto simile, quale lo spagnolo. In questi casi, dunque, bisognerebbe richiamare l'attenzione sulla microvariazione dell'articolo nelle lingue [+art].

\section{RIFERIMENTI BIBLIOGRAFICI}

Abney S. (1987), "The English noun phrase in its sentential aspect", Tesi di dottorato, Massachusetts Institute of Technology.

Aurová M., Martínez Córdoba M. (2015), "Los artículos en español: una propuesta didáctica para el nivel inicial del aprendizaje", in Revista redELE: revista electrónica de didáctica del español lengua extranjera, 27, pp. 1-24.

Belletti A. (1988), "The case of unaccusatives", in Linguistic Inquiry, 19, pp. 1-34.

Birdner B., Ward G. (1994), "Uniqueness, familiarity, and the definite article in English", in Gahl S., Dolbey A., Johnson C. (eds.), Proceedings of the Twentieth Annual Meeting of the Berkeley Linguistics Society, Berkeley Linguistics Society, Berkeley (CA), pp. 93-102.

Bosque I., Gutiérrez-Rexach J. (2008), Fundamentos de sintaxis formal, Akal, Madrid.

Bozzone Costa R. (2002), "Rassegna degli errori lessicali in testi scritti da apprendenti elementari, intermedi ed avanzati di italiano L2 (ed implicazioni didattiche)", in Linguistica e Filologia, 14, pp. 37-67.

Chiapedi N. (2010), "L'articolo italiano nell'interlingua di apprendenti sinofoni: problematiche acquisizionali e considerazioni glottodidattiche", in Italiano LinguaDue, 2, 2, pp. 53-74:

https://riviste.unimi.it/index.php/promoitals/article/view/822.

Chierchia G. (1997), "Partitives, reference to kinds and semantic variation", in Semantics and Linguistic Theory, 7, pp. 73-98.

Chierchia G. (1998), "Reference to kinds across languages", in Natural Language Semantics, 6, pp. 339-405.

Cominetti F., Tavosanis M. (in stampa), "Interferenza della L1 nell'apprendimento degli articoli in italiano L2: una ricerca sul corpus ICoN", in Borreguero Zuloaga M. (a cura di), Acquisizione e didattica dell'italiano: riflessioni teoriche, nuovi apprendenti e uno sguardo al passato, Peter Lang, Francoforte sul Meno.

Cozzani E. (2016), "Gli articoli italiani nell'interlingua di studenti ispanofoni: Analisi del sottocorpus ICoN", Tesi di laurea, Università di Pisa: https://etd.adm.unipi.it/t/etd-09062016-161608/.

Dalle Carbonare C. (2017), "L'articolo determinativo in italiano e in tedesco: un confronto attraverso l'analisi degli errori", Tesi di laurea magistrale, Università degli Studi di Padova: http://tesi.cab.unipd.it/56676/1/Chiara_Dalle_Carbonare_2017.pdf. 
De Tommaso V. (2020a), "Dalle strutture al testo. Errori in Italiano L2 di apprendenti cechi e slovacchi: osservazioni preliminari in prospettiva longitudinale", in Études romanes de Brno, 41, 1, pp. 269-286: https://doi.org/10.5817/ERB2020-1-17.

De Tommaso V. (2020b), "Penso che ci sono problemi con articoli e preposizioni': disagio linguistico nella classe di italiano L2", in Italiano LinguaDue, 12, 2, pp. 81-91: https:// riviste.unimi.it/index.php/promoitals/article/view/14974.

Donnellan K. (1966), "Reference and definite descriptions", in The Philosophical Review, 75, 3, pp. 281-304.

Doughty C. J., Williams J. (1998), "Pedagogical choices in focus on form", in Doughty C. J., Williams J. (eds.), Focus on Form in Classroom Second Language Acquisition, Cambridge University Press, Cambridge, pp. 197-261.

Ekiert M., Han Z. H. (2016), "L1-fraught difficulty: the case of L2 acquisition of English articles by Slavic speakers", in Alonso R. (a cura di), Crosslinguistic Influence in Second Language Acquisition, Multilingual Matters, Bristol, pp. 147-172.

Erguvanh E., Zimmer K. (1994), "Case marking in Turkish indefinite object constructions", in Gahl S., Dolbey A., Johnson C. (eds.), Proceedings of the Twentieth Annual Meeting of the Berkeley Linguistics Society: General Session Dedicated to the Contributions of Charles J. Fillmore, Berkeley Linguistics Society, Berkeley (CA), pp. 547-552.

Fernández Jódar R. (2006), "Análisis de errores léxicos, morfosintácticos y gráficos en la lengua escrita de los aprendices polacos de español", Tesi di dottorato, Uniwersytet im. Adama Mickiewicza w Poznaniu:

https://redined.mecd.gob.es/xmlui/bitstream/handle/11162/76535/0082010300 7276.pdf?sequence $=1$.

Fernández Jódar R. (2017), "El artículo", in Nowikow W. (ed.), Gramática contrastiva español-polaco. Lódź: Wydawnictwo Uniwersytetu Lódzkiego, 353-377.

George H. (1972), Common Errors in Language Learning, Newbury House, Rowley.

Gottardo M. G., Valentini A. (2018), "L'espressione della definitezza nell'italiano L2 di sinofoni", in Manco A. (a cura di), Le lingue extra-europee e l'italiano: aspetti didatticoacquisizionali e sociolinguistici. Atti del LI Congresso Internazionale di Studi della Società di Linguistica Italiana, Napoli, 28-30 settembre 2017), Officinaventuno, Milano, pp. 233-248:

http://www.societadilinguisticaitaliana.net/wp-

content/uploads/2019/05/Atti_SLI_LI_Congresso-Napoli-2017.pdf.

Grandi N. (2010), “Articolo", in Simone R. (a cura di), Enciclopedia dell'Italiano, Istituto dell'Enciclopedia Italiana, Roma:

https://www.treccani.it/enciclopedia/articolo_\%28Enciclopediadell $\% 27$ Italiano $\% 29 /$.

Grądzka Z. (2016), “L'interpretazione e l'uso degli articoli nella L2: Il caso dei parlanti polacco L1/italiano L2 e olandese L1/spagnolo L2", Tesi di laurea, Università di Utrecht: http://dspace.library.uu.nl/handle/1874/337090.

Greenberg J. H. (1978), Universals of human language, Stanford University Press, Stanford. Haspelmath M. (2001), "The European linguistic area: Standard Average European", in Haspelmath M., Oesterreicher W., Raible W. (eds.), Language Typology and Language Universals, Mouton De Gruyter, Berlino, pp. 1492-1510.

Hawkins J. (1978), Definiteness and Indefiniteness. A Study in Reference and Grammaticality Prediction, Croom Helms, Londra.

Hewson J. (1972), Article and Noun in English, Mouton, L'Aia. 
Holton D., Mackridge P., Philippaki-Warburton I. (2012²), Greek: A Comprehensive Grammar, Routledge, Londra-New York.

Ionin T., Ko H., Wexler K. (2004), "Article semantics in L2 acquisition: the role of specificity", in Language Acquisition, 12, 1, pp. 3-69.

Jarvis S. (2002), "Topic continuity in L2 English article use", in SSLA, 24, pp. 387-418: https://doi.org/10.1017.S0272263102003029.

Jarvis S., Grant L., Bikowskia D., Ferris D. (2003), "Exploring multiple profiles of highly rated learner compositions", in Journal of Second Language Writing, 12, 4, pp. 377-403.

Jenks P. (2018), “Articulated definiteness without articles”, in Linguistic Inquiry, 49, 3, pp. 501-536.

Kahneman D. (2011), Thinking, Fast and Slow, Farrar, Straus \& Giroux, New York.

Kahneman D., Klein G. (2009), "Conditions for intuitive expertise: a failure to disagree", in American Psychologist, 64, 6, pp. 515-526.

Kormos J. (1999), "The effect of speaker variables on the self-correction behavior of L2 learners", in System, 27, pp. 207-221.

Krifka M., Pelletier F. J., Carlson G. N., ter Meulen A., Link G., Chierchia G. (1995), "Genericity: an introduction", in Carlson G., Pelletier F. J. (eds.), The Generic Book, The University of Chicago Press, Chicago, pp. 1-124.

Krashen S. (1982), Principles and Practice in Second Language Acquisition, Pergamon, Londra.

Kucharczyk R. (2018), Nauczanie jezylków obych a dydaktyka wielojezyczności (na praylktadzie francuskiego jako drugiego jezylka obcego), Werset, Lublino.

Kyriakaki M. (2020), "Definite expressions and degrees of definiteness", in Bjorkman B. M., Currie Hall D. (eds.), Contrast and Representations in Syntax, Oxford University Press, Oxford, pp. 99-137.

Lapesa R. (1981), Historia de la lengua española, Gredos, Madrid.

Leonetti M. (1999), "El artículo", in Bosque I., Demonte, V. (eds.), Gramática descriptiva de la lengua española: Vol. 1 Sintáxis básica de las clases de palabras, Espasa, Madrid, pp. 787890.

Limonta G. (2009), “Analisi degli errori in produzioni scritte di apprendenti sinofoni”, in Italiano LinguaDue, 1, 1, pp. 29-54:

https://riviste.unimi.it/index.php/promoitals/article/view/429.

Longobardi G. (1994), "Reference and proper names: A theory of N-movement in syntax and logical form", in Linguistic Inquiry, 25, 4, pp. 609-665.

Longobardi G. (2014/1994), "Reference and proper names: A theory of N-movement in syntax and Logical Form", in Kayne R., Zanuttini R., Leu T. (eds.), An Annotated Syntax Reader: Lasting Insights and Questions, Blackwell, Hoboken, pp. 328-346.

Longobardi G. (2001), "The structure of DPs: Some principles, parameters, and problems", in Baltin M., Collins C. (eds.), The Handbook of Syntatic Theory, Blackwell, Hoboken, pp. 562-601.

Lyons C. (1999), Definiteness, Cambridge University Press, Cambridge.

Mackenzie I. (2006), Unaccusative Verbs in Romance Languages, Palgrave Macmillan, Basingstoke.

Master P. (1987), "A Cross-linguistic Interlanguage Analysis of the Acquisition of the English Article System”, Tesi di dottorato, University of California: https://www.sjsu.edu/faculty/pmaster/Master\%20Dissertation.pdf.

Master P. (1990), "Teaching the English article system as a binary system", in TESOL Quarterly, 24, 2, pp. 461-478.

Pawlik J. (2001), Selección de problemas de gramática española, Wydawnictwo Naukowe Uniwersytetu im. Adama Mickiewicza, Poznań. 
Piotrowska A., Skrzypek D. (2021), "Familiar vs. unique in a diachronic perspective. Case study of the rise of the definite article in North Germanic", in Glossa: a journal of general linguistics, 6, 1, pp. 1-21: https://doi.org/10.5334/gigl.1178.

Renzi L. (1982), "Il vero plurale dell'articolo uno", in Lingua Nostra, 43, pp. 63-68.

Renzi L. (1985), "L'articolo zero", in Franchi de Bellis A., Savoia L. M. (a cura di), Sintassi e morfologia della lingua italiana d'uso: teorie e applicazioni descrittive. Atti del XVII Congresso Internazionale di Studi della Società di Linguistica Italiana (SLI), Urbino, 11-13 settembre 1983, Bulzoni, Roma, pp. 271-288.

Renzi L. (2001), "L'articolo", in Renzi L., Salvi G., Cardinaletti A. (a cura di), Grande grammatica italiana di consultazione, vol. 1, il Mulino, Bologna, pp. 371-437.

Rizzi L. (1997), "The fine structure of the left periphery", in Haegeman L. (ed.), Elements of Grammar (Kluwer International Handbooks of Linguistics), Dordrecht, Springer, pp. 281-337: https://doi.org/10.1007/978-94-011-5420-8_7.

Rodríguez García C. (2019), "Análisis de errores en la interlengua de aprendices de ELE universitarios checos y eslovacos", Tesi di dottorato, Masarykova univerzita.

Rohlfs G. (1968), Grammatica storica della lingua italiana e dei suoi dialetti: Morfologia, Einaudi, Torino.

Rohlfs G. (1969), Grammatica storica della lingua italiana e dei suoi dialetti: Sintassi e formazione delle parole, Einaudi, Torino.

Rosi F., Berardini F. (2020), "L'acquisizione dell'italiano in Italia e in Germania: strategie lessicali per costruire la referenza", in Italiano LinguaDue, 12, 2, pp. 189-200: https://riviste.unimi.it/index.php/promoitals/article/view/15064.

Roussou A., Tsimpli I.-M. (1994), "On the interaction of case and definiteness in Modern Greek", in Philippaki-Warburton I., Nicolaidis K., Sifianou M. (eds.), Themes in Greek Linguistics, John Benjamins, Amsterdam, pp. 69-76.

Runić M. (2012), "L'uso dell'articolo italiano in apprendenti di madrelingua serbo-croata", in Ferreri S. (a cura di), Linguistica educativa. Atti del XLIV Congresso Internazionale di Studi della Società di Linguistica Italiana (SLI), Viterbo, 27-29 settembre 2010, Bulzoni, Roma, pp. 385-395.

Runić M. (2013), “The 'definite article' in the Slovene dialect of Resia”, in Pescarini D. (a cura di), Quaderni di lavoro ASIt, 16, Atti della XVIII Giornata di Dialettologia, Unipress, Padova, pp. 91-106.

Russell B. (1905), "On denoting", in Mind, 14, pp. 479-493: https://www.jstor.org/stable/2248381.

Schmitt C., Munn A. (2002), "The syntax and semantics of bare arguments in Brazilian Portuguese", in Pica P., Rooryck J. (eds.), Linguistic Variation Yearbook 2, John Benjamins, Amsterdam-Philadelphia, pp. 185-216.

Schwartz F. (2009), "Two types of definites in natural language", Tesi di dottorato, University of Massachusetts:

https://scholarworks.umass.edu/open_access_dissertations/122/.

Serianni L. (2006), Grammatica italiana. Italiano comune e lingua letteraria, UTET, Torino.

Stanič D. (2017), "Analysis of errors in written production of students of Italian as a foreign language in the university context", in Journal for Foreign Languages, 9, 1, pp. 255-285:

https://doi.org/10.4312/vestnik.9.255-285.

Suñer M. (1982), Syntax and Semantics of Spanish Presentational Sentence-Types, Georgetown University Press, Washington.

Sussex R., Cubberley P. (2006), The Slavic Languages, Cambridge University Press, New York. 
Škevin I., Maroević A. (2014), "L'approccio comparativo nella didattica dell'italiano ai croati: articolo e preposizione", in Grammatica e Didattica. Atti delle Giornate di "Linguistica e Didattica", Padova 25-26 febbraio 2014, pp. 123-148.

Szabolcsi A. (1983), "The possessor that ran away from home”, in The Linguistic Review, 3, pp. $89-102$.

Szwedek A. (1976), "Pronouns as articles?", in Papers and Studies in Contrastive Linguistics, 4, pp. 265-271.

Testa M. (2019). "The acquisition of L3 Spanish articles: What can be learned from a simple linear regression analysis?", in Research in Corpus Linguistics, 7, pp. 84-112.

Testa M. (2021), "Una heurística para la enseñanza de los artículos españoles", in Itinerarios. Revista de estudios lingüisticos, literarios, históricos y antropológicos, 33, pp. 205224: https://doi.org/10.7311/itinerarios.33.2021.12.

Trenkić D. (2009), Accounting for patterns of article omissions and substitutions in second language production", in Hawkins R., García Mayo M. (eds.) Second language acquisition of articles: Empirical findings and theoretical implications, John Benjamins, Amsterdam, pp. 115-143.

Tversky A., Kahneman D. (1974), "Judgment under uncertainty: heuristics and biases", in Science, 185, pp. 1124-1131.

van der Auwera J. (1998), "Conclusion", in van der Auwera J. (ed.), Adverbial Constructions in the Languages of Europe, Mouton de Gruyter, Berlino, pp. 813-836.

Viviani A. (2011), "Articolo [prontuario]", in Simone R. (ed.), Enciclopedia dell'Italiano, Istituto dell'Enciclopedia Italiana, Roma:

https://www.treccani.it/enciclopedia/articolo-prontuario_\%28Enciclopediadell $\% 27$ Italiano $\% 29 /$.

Wilson D., Sperber D. (2012), "Linguistic form and relevance", in Wilson D., Sperber D. (eds.), Meaning and Relevance, Cambridge University Press, Cambridge, pp. 149-168.

Zgrinskić R. (2019), “Analisi dell”errore: Errori in temi di italiano come lingua seconda e studio dell'interferenza slovena", Tesi di laurea magistrale, Univerza v Ljubljani: https:/ / repozitorij.uni-lj.si/Dokument.php?id=124490\&lang=slv. 